\title{
Freehand Pedicle Screw Insertion in Spondylitis Tuberculosis Kyphosis Correction Using Cantilever Method: A Breach Rate Analysis of 168 Consecutive Screws
}

\author{
Didik Librianto $\mathbb{D}^{\prime}$, Ifran Saleh ${ }^{2}$, Fachrisal Ipang $\mathbb{D}^{\prime}$, Dina Aprilya $\mathbb{D}^{2}$ \\ 'Orthopedic Spine Surgery, Fatmawati General Hospital, Jakarta, Indonesia; ${ }^{2}$ Department of Orthopedic and Traumatology, Faculty of Medicine \\ Universitas Indonesia, Jakarta, Indonesia
}

Correspondence: Dina Aprilya, Department of Orthopedic and Traumatology, Faculty of Medicine Universitas Indonesia, Prof. Soelarto building, Ist floor, RS Fatmawati Street, Jakarta, 12430, Indonesia, Tel +62 89655106136, Fax +62 21-7660616, Email dina.cia.aprilya@gmail.com

Background: The cantilever method is a standard for two-dimensional deformity correction, as in spondylitis tuberculosis kyphotic deformity. An accurate and secured pedicle screw placement as part of the correction tools is essential to accommodate reduction while preventing screw-related complications. Many literatures have described the pedicle screw misplacement in cases with "normal" bone quality (ie, scoliosis, Scheuermann's kyphosis, ankylosing spondylitis, trauma) or in the obviously abnormal bone such as osteoporosis. However, to our knowledge, the pedicle screw accuracy in cases of deformity correction of tuberculous kyphosis was not previously reported.

Methods: This is a retrospective study of 168 pedicle screws in 14 consecutive cases of spondylitis tuberculosis with kyphotic deformity. The cantilever reduction method with freehand screw insertion technique was done in all cases to correct the deformity. Postoperative computed tomography (CT) evaluation was done to evaluate screw position and breach rates.

Results: Among the 168 screws, accurate pedicle screw placement was accomplished in 39.3\% screws (Gertzbein-Robbins Grade A). The overall breach rate was $61.9 \%$, which was most commonly occurred on the segment proximal to the apex of the deformity $(\mathrm{p}=0.001)$. The lateral breach was more common than the medial breach $(52.3 \% \mathrm{vs} 7.7 \%)$. The pedicle screw on the thoracal region has a greater breach incidence than those on the lumbar region especially those on T9, T10, and T11. There was no injury to the surrounding neurovascular and pleural structures. No revision surgeries were required.

Conclusion: Freehand pedicle screw insertion in spondylitis tuberculosis kyphotic reduction has proved to be safe. However, the accuracy should be improved to prevent long-term screw-related complications.

Keywords: thoracolumbar spine, spondylitis tuberculosis, kyphosis, pedicle screws, freehand technique, cantilever method

\section{Introduction}

The progressive anterior column destruction in spondylitis tuberculosis infection often results in kyphotic deformity. Together with instability, large abscess, or neurological deficit, this disfigurement often necessitates surgical procedures to decompress the nerve, stabilize the spine, and correct the deformity. ${ }^{1,2}$ The cantilever method is still a gold standard to correct the sagittal plane deformity that has proved as an effective and safe method to correct the kyphotic deformity in spondylitis tuberculosis cases. ${ }^{3,4}$ It takes the advantage of the pedicle screw-rod system's multidimensional control, which is favorable for deformity correction.

The pedicle screw-rod system had gained popularity since its first introduction 71 years ago due to the safety and biomechanical benefits compared to its predecessors. The screw can be inserted either with the freehand technique or assisted with navigation technology. The freehand technique is still the most common technique used worldwide especially in developing countries where the navigation technology is either unavailable or unaffordable. This technique relies on the appreciation of spinal anatomy from preoperative imaging and intraoperative landmarks. ${ }^{5}$ 
There have been many studies illustrating the screw accuracy either using freehand technique or with assisted tools in the different institutional practices. Variation of anatomical challenges such as in spinal deformity cases has also been widely studied. However, in the case of spondylitis tuberculosis, the deformity along with the infectious bone itself creates a challenge in inserting and securing the fixation safely and accurately while performing the reduction. While screw misplacement can be catastrophic, to our knowledge, no literature provides evidence of pedicle screw misplacement in spondylitis tuberculosis kyphotic correction surgery. The aim of this study is to evaluate the accuracy of pedicle screw insertion in spondylitis tuberculosis kyphosis correction using a freehand technique. ${ }^{5-7}$

\section{Materials and Methods}

This is a retrospective study of kyphotic spondylitis tuberculosis (Stb) patients conducted at Fatmawati General Hospital, Jakarta, Indonesia, through the year 2020-2021. Cervical spondylitis tuberculosis was excluded due to the different techniques of fixation. Other spinal deformities (ie, Scoliosis de Novo), previous spinal instrumentation, spinal tumor, trauma, and pyogenic infection, were not included in the study. Among 32 eligible cases, 10 patients failed to fulfill the postoperative CT scan and 8 patients refused to be involved as research subjects. Finally, 14 cases with a total of 168 pedicle screws were included in this study. Plain radiographs were taken before and after surgery to measure the sagittal Cobb angle. Intraoperative radiography and intraoperative neuromonitoring (IONM) were performed in all subjects. Somatosensory evoked potentials (SSEP) and spinal motor evoked potential (MEP) were recorded before the incision (baseline), after screw insertion, and after deformity correction (final). The intraoperative signals were compared to the baseline signals to indicate nerve injury or improvement.

The reduction technique consisted of cantilever bending method by utilizing pedicle screw-rod system. The pedicle screws were inserted using freehand technique. The pre-bend rods were secured to pedicle screws sequentially from proximal towards the apex of deformity and vertebrae below it. Within the first month postoperatively, the pedicle screw position was evaluated with computed tomography (CT) scan and was measured using iQ-LITE 3.0.0 Viewer ${ }^{\circledR}$ by two independent observers ((orthopedic spine surgeon (F.C) and general orthopedic surgeon (D.A)). Neurological complications due to screw misplacement requiring screw revision were recorded.

\section{Pedicle Screw Insertion}

The screw insertion is performed using anatomical landmark to identify the entry point. Screw entry point is prepared by removing the overlying soft tissue. A pilot hole is made by an awl The hole was probed and assessed for a breach. The hole was tapped and a pin was inserted to the preliminary hole. The trajectory was checked by the intraoperative radiograph and the hole was re-palpated to detect any breach of pedicle walls (medial, lateral, anterior, superior, and inferior). Screws with appropriate size and length were placed in the screw-hole with good trajectory.

\section{CT-Scan Evaluation}

Postoperatively, patients underwent CT scans to assess the accuracy of screw placement according to GertzbeinRobbins's classification (graded from A to E based on the extent of cortical breaching) as shown in Figure 1. Grade $\mathrm{A}$ is described when the screw is fully intrapedicular without breaching the cortex. Grade B-E is screw position exceeding the pedicle cortex for $<2 \mathrm{~mm}, 2-4 \mathrm{~mm}, 4-6 \mathrm{~mm}$, and $>6 \mathrm{~mm}$, respectively. In addition, screw accuracy was categorized using Karagoz-Guzey modification scale to differentiate the direction and magnitude of the violating screw either medial (M), lateral (L), or anterior (A) direction. The magnitude of penetration is described as a number following the screw direction: 1) $<2 \mathrm{~mm}$; 2) $2-4 \mathrm{~mm}$; and 3) $>4 \mathrm{~mm}$ or wholly outside the canal. ${ }^{8-10}$ Blinding was done as the measurement was performed by two independent observers who did not perform the surgery and did in the slice with the largest screw deviation from the pedicle.

The statistical testing was performed using IBM SPSS Statistic 25 with parametric paired $t$-test, non-parametric statistics using Mann-Whitney $U$, and proportion data using Chi-Square and Fisher's exact test. A $p<0.05$ was considered statistically significant. 


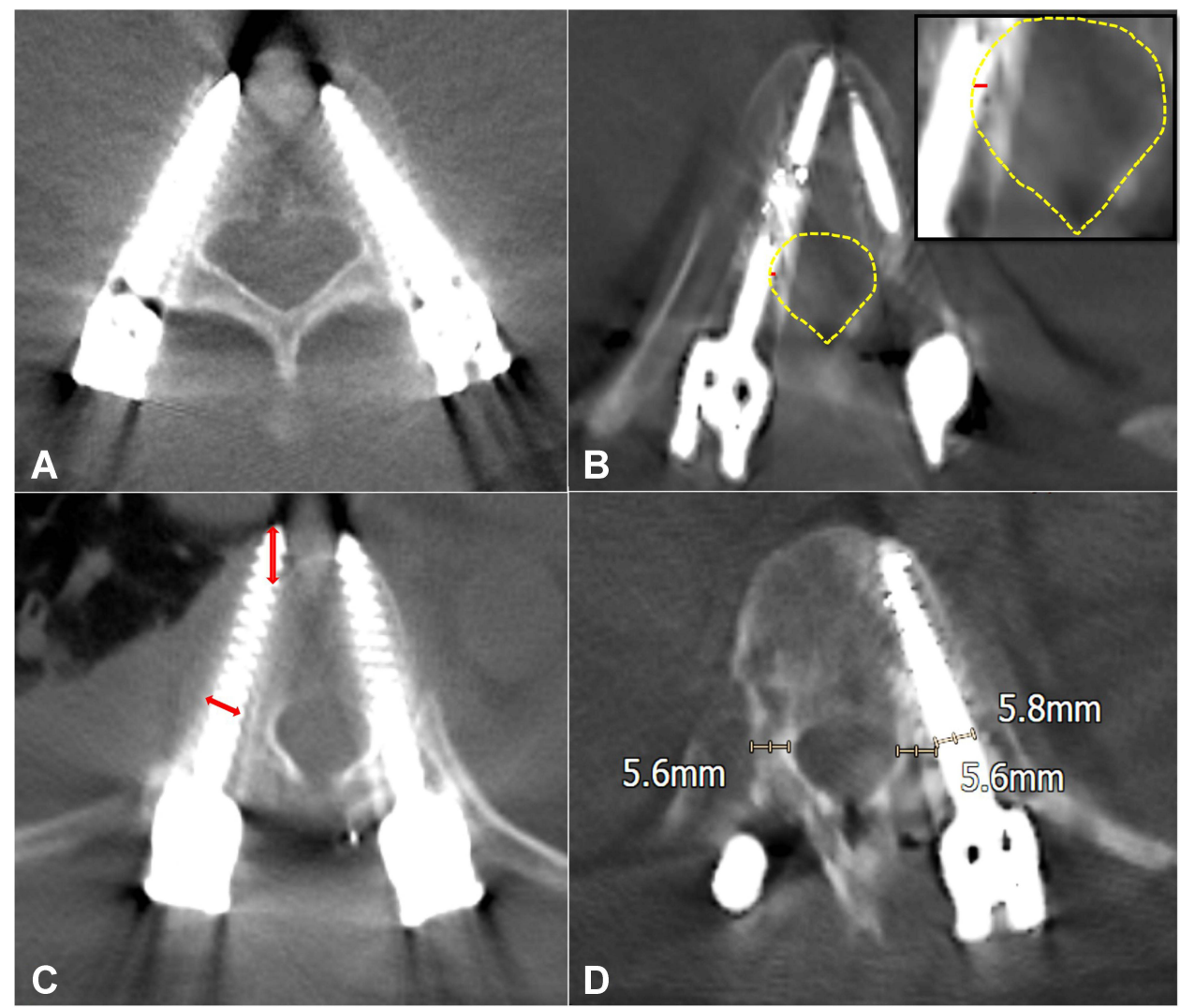

Figure I CT-image evaluation for pedicle screw breach. (A) Gertzbein-Robbins Grade A. The entire screw is intrapedicular. (B) Gertzbein-Robbins Grade B and medial breach grade I (red line: part of screw outside the cortex $<2 \mathrm{~mm}$; yellow line: medial cortex). (C) Gertzbein-Robbins Grade D with lateral and anterior breach (red arrows: Part of screw outside the cortex 4-6 mm). (D) Gertzbein-Robbins Grade E with lateral breaching. Noted that almost entire screw diameter is outside the pedicle.

\section{Results}

A total of 168 screws from 14 spondylitis tuberculosis patients were evaluated. The male-to-female ratio was 5:9. The mean age was $33.43 \pm 10.797$ years and the mean body mass index (BMI) was $21.96 \pm 2.280 \mathrm{~kg} / \mathrm{m}^{2}$. The kyphotic Cobb angle was $39.36 \pm 12.438^{\circ}$ with apex that lies within the thoracal and thoracolumbar region. The segment proximal to the apex of deformity involved 80 screws that placed in thoracal spine and 4 screws in L1 spine.

Overall, the breach rate was $61.9 \%$ which was significantly higher on the segment proximal to apex compared to the distal segment (Table 1). Among the breach incidence, most common violated site was on thoracal spine T9, T10, and T11 (each incidence was $13.5 \%$ among all breached screws) (Figure 2). Perfect screw trajectory was recorded in $39.3 \%$ screws (Figure 3). While the remaining were further classified according to the breach direction. Anterior breach occurred in $14.2 \%$ screws without significant difference between the proximal and the distal segment. Lateral breach occurred in $52.3 \%$ screws and medial breach occurred in $7.7 \%$ screws. The rate of lateral and medial breach was significantly higher in the proximal segment $(p<0.001)$. There were no cranial or caudal breach occurred. There were no grade III breach with medial direction (Table 2).

The breach length outside the pedicle and vertebral body cortex were measured. Among the anterior and medial breach screws, the length difference between the proximal and distal segment failed to reach statistical significance ( $p$ value 0.589 and 0.268 , respectively). Meanwhile, the lateral breach length on the proximal screws was significantly longer.

Both post correction clinical neurological status and IONM showed improvement compared to the preoperative status (35.7\% and 21.4\%, respectively) (Figure 4). There was a significant improvement of final intraoperative SSEP and MEP compared to baseline ( $p$ value $<0.001$ and 0.003 , respectively). The surgery was also successfully corrected the deformity as shown in the reduction of Cobb's angle from $39.36 \pm 12.438$ to $16.35 \pm 7.692(p<0.001)$. 
Table I Breach Rate Analysis on Screws Placed in the Proximal and Distal Segment of the Kyphosis

\begin{tabular}{|c|c|c|c|c|}
\hline & Proximal Segment (\%) & Distal Segment (\%) & Total (\%) & $P$ value (Proximal vs Distal) \\
\hline Breach & 75 & 48.8 & 61.9 & $0.00 I^{\mathrm{a}}$ \\
\hline Gertzbein-Robbins Classification & & & & $<0.001^{\mathrm{b}}$ \\
\hline A & 25 & 53.6 & 39.3 & \\
\hline B & 26.2 & 32.1 & 29.2 & \\
\hline C & 28.6 & 9.5 & 19 & \\
\hline D & 14.3 & 3.6 & 8.9 & \\
\hline$E$ & 6 & 1.2 & 3.6 & \\
\hline Anterior Breach & & & 14.2 & $0.079^{b}$ \\
\hline Al & 3.6 & 7.1 & 5.4 & \\
\hline $\mathrm{A} 2$ & 7.1 & 2.4 & 4.8 & \\
\hline A3 & 7.1 & 1.2 & 4.2 & \\
\hline Axial Breach & & & & $<0.00 \mathrm{I}^{\mathrm{b}}$ \\
\hline Lateral Breach & & & 52.3 & \\
\hline LI & 15.5 & 27.4 & 21.4 & \\
\hline L2 & 31.0 & 11.9 & 21.4 & \\
\hline L3 & 16.7 & 2.4 & 9.5 & \\
\hline Medial Breach & & & 7.7 & \\
\hline MI & 10.7 & 3.6 & 7.1 & \\
\hline M2 & 1.2 & 0 & 0.6 & \\
\hline M3 & 0 & 0 & 0 & \\
\hline \multicolumn{5}{|l|}{ Sagittal Breach } \\
\hline Cranial & 0 & 0 & 0 & \\
\hline Caudal & 0 & 0 & 0 & \\
\hline
\end{tabular}

Notes: ${ }^{\text {Pearson }}$ Chi-square. ${ }^{\mathrm{b}}$ Fisher's exact test.

There was no intraoperative dural tear, vascular or neurological injury that required screw revision. Within 1-month follow-up, there was no new emerging neurological deficit or new neurological impingement, and so as other immediate perioperative complications such as injury to major vessel, pleura, peritoneal, or urinary tract.

\section{Breach Incidence}

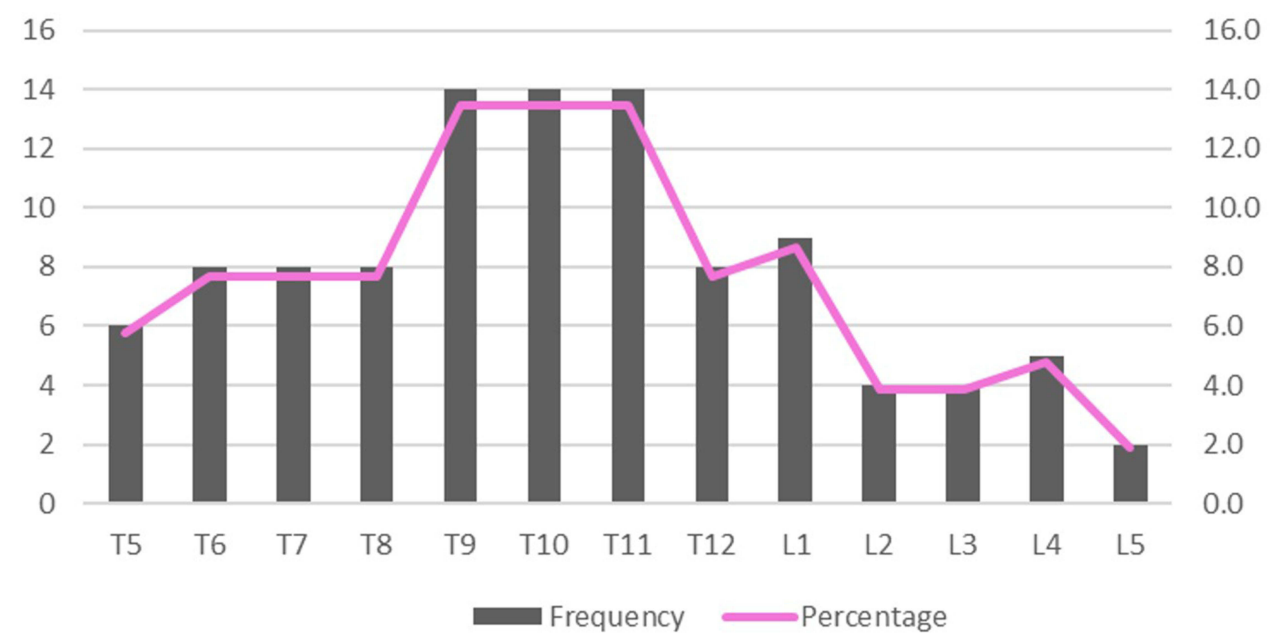

Figure 2 Distribution of breach screw in relation to pedicle level. The most common sites for breach were T9, TI0, and TII (I3.5\%). The LI was the most common sites for breach among the lumbar spine $(8.7 \%$ of all pedicle levels). 


\section{Gertzbein and Robbins Classification}

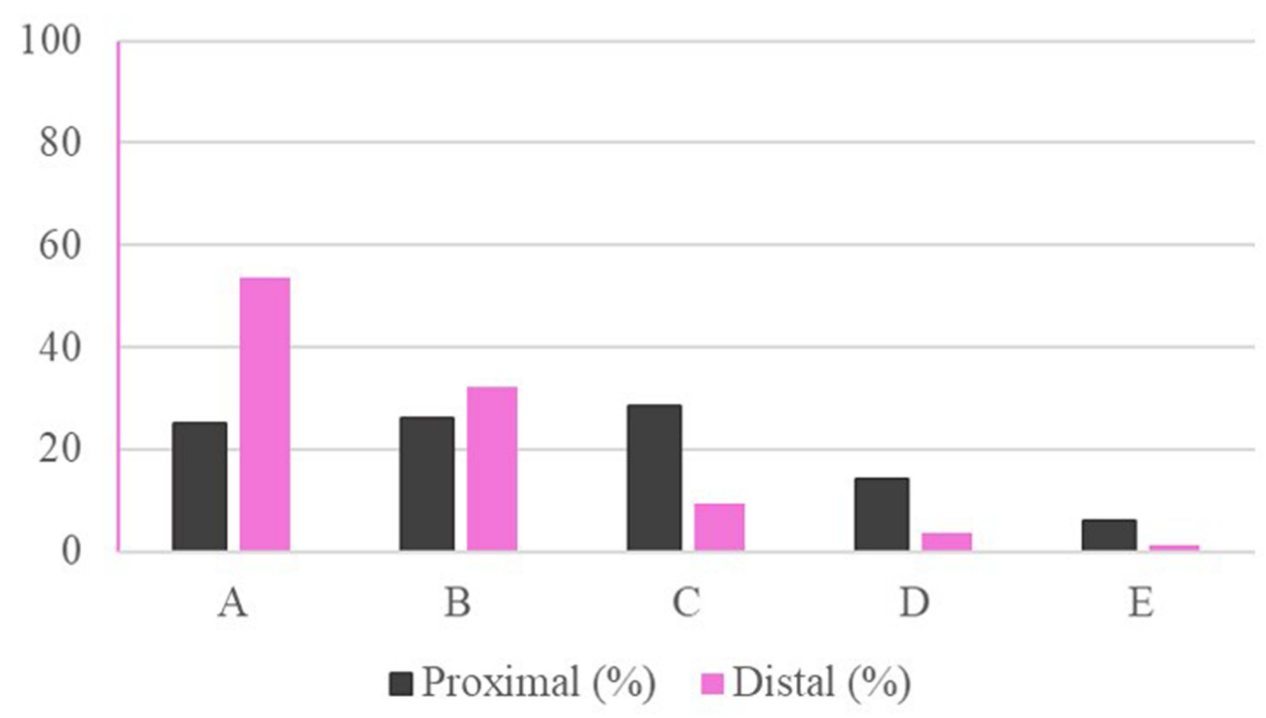

Figure 3 Distribution of breach grading according to Gertzbein and Robbins in relation to the screw segments toward the apex.

\section{Discussion}

The cantilever reduction method is still the gold standard to correct two-dimensional sagittal deformities such as kyphotic deformity. A cantilever means any projecting structure that is fixed at one end and carries a load along its length until the opposite end. Pedicle screws can act as the cantilever in the spinal fixation construct but must be able to withstand deformation under physiological loads. ${ }^{3,11}$

Compared to the earlier fixation method such as hook and rod system, the pedicle screw-rod system offers more structural rigidity that allows stability needed for spinal fusion. Moreover, it allows a three-column control that is necessary for deformity correction. Despite its potential benefit, pedicle screw insertion is not risk-free. The screwrelated complications such as spinal canal violation, pedicle fracture, nerve root compression, and vascular lesion are still haunting although the inserting procedure has been standardized. Several methods have been developed to improve the accuracy and safety such as guide pins, intraoperative radiography, intraoperative neuro-monitoring guided, until the most recently 3D navigation guided such as O-arm, intraoperative CT scan, and robotic-assisted pedicle screw placement. ${ }^{6,7,12}$

In terms of cantilever devices, the pedicle screw must be able to withstand the force applied during the correction. They shall be placed securely in a good trajectory within the compact structure of the vertebrae - intrapedicularly towards the center of the vertebral body. To achieve the perfect position, the screw insertion technique is standardized and differs according to the structural characteristic of each vertebra. For instance, the pedicle on the thoracal spine is usually narrow and inconsistent in shape compared to the lumbar pedicle. Thus, the anatomical landmark for screw entry is challenging in the thoracal spine. ${ }^{3,13}$

In a systematic review by Gelalis et $\mathrm{al}^{14}$ several factors might affect the screw accuracy regardless of the navigation (or freehand) technique for screw placement: The patient demographic characteristics, indications for surgery, surgeons'

Table 2 Length of the Cortical Breach

\begin{tabular}{|l|l|l|l|}
\hline Breach Length (mm) & Proximal (Median, Range) & Distal (Median, Range) & $\boldsymbol{p}$ value \\
\hline Anterior Breach & $0(0-6.9)$ & $0(0-7.5)$ & 0.589 \\
Medial Breach & $1.4(0.6-3.3)$ & $1.6(1.3-1.9)$ & 0.268 \\
Lateral Breach & $3.3(0.6-7.7)$ & $2.1(0.6-6.9)$ & $<0.001$ \\
\hline
\end{tabular}




\section{Frankel}

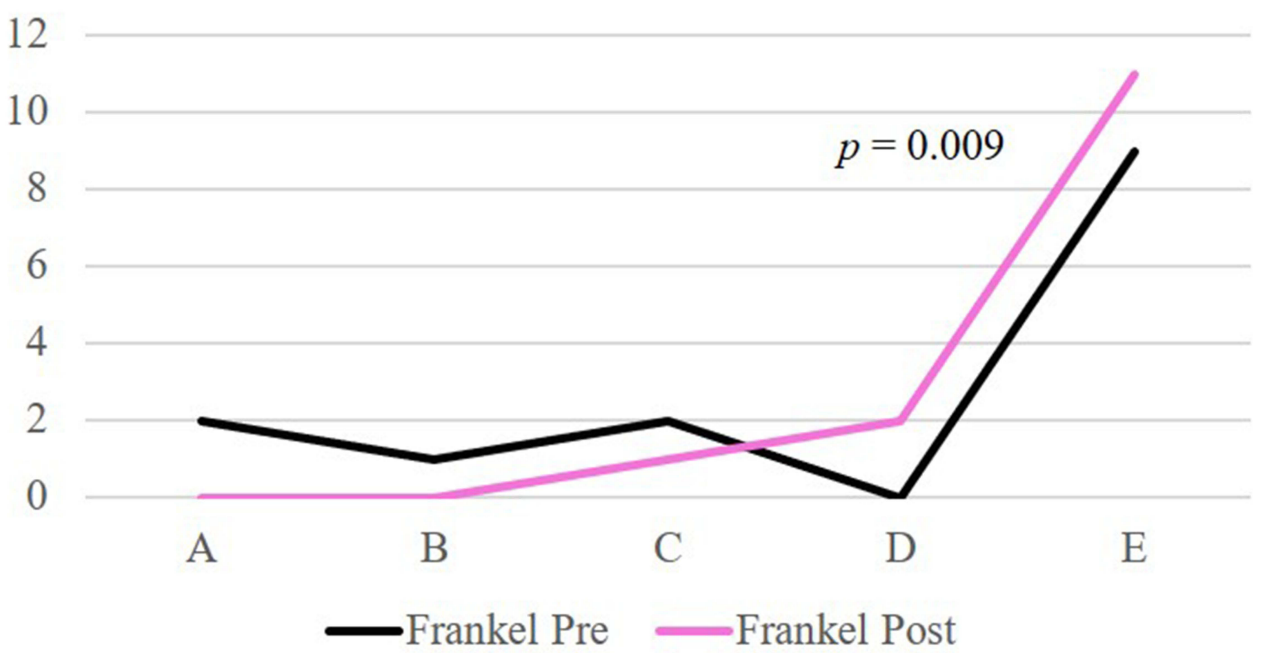

Figure 4 Distribution and comparison of pre- and post-operative Frankel. Post operatively, there was a significant improvement of neurological status.

skills, the complexity of the surgery, and screw/pedicle dimensions. ${ }^{14}$ The freehand techniques have been noted to have a significant learning curve. In a study by Gertzbein et $\mathrm{al}^{10}$ the accuracy rates were increasing in the last $25 \%$ of placed screws compared to the accuracy of the entire study. Obviously, regardless of the insertion technique, the surgeon's experience determines the ability to accurately place pedicle screws. In our study, the attending surgeon had a 15-year experience as a spine surgeon with lots of experience in deformity correction as well as the endemic tuberculosis infection in the spine. In our previous publication, we have evaluated the screw accuracy for scoliosis correction and in this study, we particularly aim to evaluate the accuracy of screw placement for deformity correction in a more challenging bone condition. ${ }^{15}$

Several studies have evaluated the accuracy of freehand pedicle screw insertion. Puvenaserajah et $\mathrm{al}^{5}$ reviews the reported case series from 2004 to 2014. In their review, the accuracy rates ranged from $71.9 \%$ to $98.3 \%$, with thoracal screws having a greater incidence of breaches. Other articles reported the screw misplacement rates in the thoracal spine with the rate of not more than $30 \%$, which differs significantly from the rate on the lumbar region. ${ }^{13,16}$ Furthermore, more breaches were the result of lateral malposition. ${ }^{8,13}$ Similar to other studies, we also found that the lateral breach is significantly higher than the medial breach. As medial cortical breaching can directly violate the spinal cord, in a freehand technique, the surgeon will tend to avoid medial direction during screw placement. Moreover, the thicker medial pedicular wall may also take part. Breaching may also be attributed to screw-pedicle dimension disproportion in case of too small pedicle to accept the full screw diameter. In our study, this case occurred more often than the missed trajectory. ${ }^{7,15,16}$

Grades A and B in Gertzbein-Robbins's classification are clinically acceptable, whereas screws graded C, D, and E have a significant deviation from the intended trajectory and potentially lead to complications. ${ }^{8,12}$ Among all screws, there were $69.1 \%$ screws were Gertzbein-Robbins Grades A and B, and 19\%, 8.9\%, and 3.6\% for Grade CE consequently. The concept of a safe zone as purposed by Gertzbein et al ${ }^{10}$ is the $4 \mathrm{~mm}$ of the allowable medial breach within the lower thoracic and lumbar spine that is considered as the size of epidural space and subarachnoid space of each vertebra level. In the main and upper thoracic region, this margin is reduced to $2 \mathrm{~mm}$. However, a more realistic measure would be the reported revision rates or patient morbidity, which are not always reported together with the accuracy rates among the reported cases in the literature. ${ }^{5,17}$

In our study, the breach rate seems slightly higher compared to other studies that evaluate the accuracy in otherwise healthy bones. However, there were no clinical morbidities and revision surgeries. This result supports other literature in 
which the incidence of neurological complications related to screw misplacement is extremely low. Indeed, before the screw placement, several steps were taken to prevent breaches such as pinning, palpating all pedicle walls with the probe, and intraoperative radiographs. It is likely that the misplaced screws did occur despite the properly made pilot hole. This may be caused partly to the self-tapping and self-drilling nature of the current pedicle screw system, which potentially contributes to the misplacement. ${ }^{16}$

Moreover, the relatively high incidence of breaching screws (30.9\%) in our study may also be influenced by the poor bone quality resulting from the bed-ridden tendency of the patient with reduced activity due to the neurological deficit. Up to $62 \%$ of osteoporotic spine fixation by pedicle screw instrumentation faced screw-related complications such as screw loosening, migration, or pullout. This insufficiency to withstand forceful events during the cantilever maneuver may potentially contribute to breach incidence, especially in areas subjected to greater force such as in the proximal segment of the construct. ${ }^{18}$ Accordingly, we found that the majority of breaches occurred on the proximal segment.

Image-guided navigation has been proven to reduce the breach rate. The development of intraoperative threedimensional imaging (stereotactic) as well as robotic surgeries enables surgeons to insert the screw in a better 3-D fashion. Even in the uncertainty of thoracic spine anatomy, CT navigation had proven to have a better breach rate (4.5\%) while compared with fluoroscopy-guided $(21.0 \%)$. In such a way, navigation-guided pedicle screw insertion is recommended in patients with a significant deformity particularly when involving upper to mid-thoracic instrumentation. Unfortunately, this technology is still not widely used due to financial issues. Thus, the freehand technique is still considered a standard procedure and its accuracy should be increased with experience, preoperative measurements, along with intraoperative findings, measurements, and image intensifier guidance. ${ }^{5,19}$

\section{Conclusion}

The freehand technique for pedicular screw insertion has demonstrated reasonable results in the past few decades. Its application as part of the cantilever reduction method for spondylitis tuberculosis is proven to be safe. Although the breach rate on CT-imaging does not correlate to the clinical picture in our study, attempts to increase pedicle screw accuracy should be implemented especially in cases with poor bone quality. A larger study with a longer follow-up time is needed to evaluate the long-term screw-related complication. Furthermore, a biomechanical study is also essential to predict the screw misplacement in deformity correction surgery of patients with poor bone quality.

\section{Data Sharing Statement}

There are no other supporting data.

\section{Ethics Approval and Consent to Participate}

This research had already been approved by ethical committee in Fatmawati General Hospital Jakarta. The written informed consent has been obtained from all participants. The privacy and personal identity information of all participants were protected in accordance with the Declaration of Helsinki.

\section{Consent for Publication}

My manuscript does not contain any individual person data. The co-authors consented to the publication of this article.

\section{Funding}

This research did not receive any specific grant from funding agencies in the public, commercial, or not-for-profit sectors.

\section{Disclosure}

The authors declare that they have no conflicts of interest for this work. 


\section{References}

1. Rajasekaran S, Soundararajan DCR, Shetty AP, Kanna RM. Spinal tuberculosis: current concepts. Glob Spine J. 2018;8(4_suppl):96S-108S. doi:10.1177/2192568218769053

2. Tuli SM. Severe kyphotic deformity in tuberculosis of the spine. Int Orthop. 1995;19(5):327-331. doi:10.1007/BF00181121

3. Librianto D, Aprilya D. Cantilever method for severe kyphotic deformity correction in spondylitis tuberculosis: a technical note and literature review. Ann Med Surg. 2021;69:102764. doi:10.1016/j.amsu.2021.102764

4. Senkoylu A, Cetinkaya M. Correction manoeuvres in the surgical treatment of spinal deformities. EFORT Open Rev. 2017;2(5):135-140. doi:10.1302/2058-5241.2.170002

5. Puvanesarajah V, Liauw JA, Lo SF, Lina IA, Witham TF. Techniques and accuracy of thoracolumbar pedicle screw placement. World J Orthop. 2014;5(2):112-123. doi:10.5312/wjo.v5.i2.112

6. Qian B-P, Zhang Y-P, Qiao M, Qiu Y, Mao S-H. Accuracy of freehand pedicle screw placement in surgical correction of thoracolumbar kyphosis secondary to ankylosing spondylitis: a computed tomography investigation of 2314 consecutive screws. World Neurosurg. 2018;116:e850-e855. doi:10.1016/j.wneu.2018.05.116

7. Min WK, Na SB, Jang JA. Accuracy of thoracic pedicle screw placement using freehand technique and triggered EMG in adolescent idiopathic scoliosis: is it different between concave and convex side? J Orthop Surg. 2018;26(2):1-6. doi:10.1177/2309499018784975

8. Gruenberg M, Petracchi M, Valacco M, Solá C. The influence of anatomy (normal versus scoliosis) on the free-hand placement of pedicle screws: is misplacement more frequent in patients with anatomical deformity? Evid Based Spine Care J. 2010;1(02):11-17. doi:10.1055/s-0028-1100909

9. Guzey FK, Emel E, Hakan Seyithanoglu M, et al. Accuracy of pedicle screw placement for upper and middle thoracic pathologies without coronal plane spinal deformity using conventional methods. J Spinal Disord Tech. 2006;19(6):436-441. doi:10.1097/00024720-200608000-00011

10. Gertzbein SD, Robbins SE. Accuracy of pedicle screw placement in vivo. Spine (Phila Pa 1976). 1990;16(1):11-14. doi:10.1097/00007632199001000-00004

11. Rajpal S, Resnick DK. Rod cantilever techniques. Neurosurgery. 2008;63(3SUPPL):157-162. doi:10.1227/01.NEU.0000325767.45588.A2

12. Solomiichuk V, Fleischhammer J, Molliqaj G, et al. Robotic versus fluoroscopy-guided pedicle screw insertion for metastatic spinal disease: a matched-cohort comparison. Neurosurg Focus. 2017;42(5):E13. doi:10.3171/2017.3.FOCUS1710

13. Mattei TA, Meneses MS, Milano JB, Ramina R. "Free-hand" technique for thoracolumbar pedicle screw instrumentation: critical appraisal of current "state-of-art". Neurol India. 2009;57(6):715-721. doi:10.4103/0028-3886.59465

14. Gelalis ID, Paschos NK, Pakos EE, Politis AN. Accuracy of pedicle screw placement: a systematic review of prospective in vivo studies comparing free hand, fluoroscopy guidance and navigation techniques. Eur Spine J. 2012;21:247-255. doi:10.1007/s00586-011-2011-3

15. Librianto D, Saleh I, Saleh F, Utami WS, Hutami WD. Breach rate analysis of pedicle screw instrumentation using free-hand technique in the surgical correction of adolescent idiopathic scoliosis. J Orthop Case Rep. 2021;11(1):38-44. doi:10.13107/jocr.2021.v11.i01.1956

16. Parker SL, McGirt MJ, Farber SH, et al. Accuracy of free-hand pedicle screws in the thoracic and lumbar spine: analysis of 6816 consecutive screws. Neurosurgery. 2011;68(1):170-178. doi:10.1227/NEU.0b013e3181fdfaf4

17. Belmont J, Klemme WR, Dhawan A, Polly J. In vivo accuracy of thoracic pedicle screws. Spine (Phila Pa 1976). 2001;26(21):2340-2346. doi:10.1097/00007632-200111010-00010

18. Tandon V, Kalidindi KKV, Pacha S, Bhat MR. A prospective study on the feasibility, safety, and efficacy of a modified technique to augment the strength of pedicle screw in osteoporotic spine. Asian Spine J. 2020;14(3):357-363. doi:10.31616/ASJ.2019.0211

19. Quraishi NA, Lewis SJ, Kelleher MO, Sarjeant R, Rampersaud YR, Fehlings MG. Intraoperative multimodality monitoring in adult spinal deformity: analysis of a prospective series of one hundred two cases with independent evaluation. Spine (Phila Pa 1976). 2009;34 (14):1504-1512. doi:10.1097/BRS.0b013e3181a87b66

Orthopedic Research and Reviews

Dovepress

\section{Publish your work in this journal}

Orthopedic Research and Reviews is an international, peer-reviewed, open access journal that focusing on the patho-physiology of the musculoskeletal system, trauma, surgery and other corrective interventions to restore mobility and function. Advances in new technologies, materials, techniques and pharmacological agents are particularly welcome. The manuscript management system is completely online and includes a very quick and fair peer-review system, which is all easy to use. Visit http://www.dovepress.com/testimonials.php to read real quotes from published authors.

Submit your manuscript here: https://www.dovepress.com/orthopedic-research-and-reviews-journal 